\title{
GMR
}

\section{A novel splice variant of the bovine $G A L N T L 5$ gene identified in Chinese Holstein bull testis tissue and its mRNA expression}

\author{
H. Li ${ }^{1,2 *}$, Q. Jiang ${ }^{1 *}$, Z.H. Ju' ${ }^{1}$, J.M. Huang ${ }^{1}$, X.G. Wang ${ }^{1}$, C.H. Yang ${ }^{1}$, \\ Y. Sun ${ }^{1}$, G.W. Yang ${ }^{2}$, J.F. Zhong ${ }^{1}$ and C.F. Wang ${ }^{1}$ \\ ${ }^{1}$ Dairy Cattle Research Center, Shandong Academy of Agricultural Sciences, \\ Jinan, Shandong, China \\ ${ }^{2}$ College of Life Science, Shandong Normal University, Jinan, China \\ *These authors contributed equally to this study. \\ Corresponding authors: Z.H. Ju / C.F. Wang \\ E-mail: juzhihua283@163.com / wangcf1967@163.com
}

Genet. Mol. Res. 15 (2): gmr.15027723

Received September 24, 2015

Accepted December 9, 2015

Published April 27, 2016

DOI http://dx.doi.org/10.4238/gmr.15027723

\begin{abstract}
The polypeptide $\mathrm{N}$-acetylgalactosaminyltransferase-like protein 5 (GALNTL5) is a newly identified protein that is specifically expressed in testis tissue and participates in spermatogenesis. In this study, we characterized a novel bovine GALNTL5 splice variant, designated as GALNTL5-AS, by using real-time polymerase chain reaction (RT-PCR) and clone sequencing methods. The novel GALNTL5 isoform was derived from the complete transcript, GALNTL5complete, via alternative splicing (AS). The pattern of the splice variant was exon skipping. Bovine GALNTL5 transcripts were expressed in the testis, as demonstrated by RT-PCR. The expression levels of both transcripts were higher in adult testes than in calf testes $(\mathrm{P}<0.05)$. In addition, prediction analysis showed that the GALNTL5-AS transcript only encoded 122 amino acids and lost its glycosyltransferase 1 and $\mathrm{Gal} /$ GalNAc-T motifs, which may result in a dysfunctional protein compared with the predominant transcript GALNTL5-complete.
\end{abstract}


This study improves our understanding of the bovine GALNTL5 gene function during bull sperm formation.

Key words: Alternative splicing; Chinese Holstein bull; Testis; GALNTL5 gene; Quantitative real-time polymerase chain reaction

\section{INTRODUCTION}

The polypeptide N-acetylgalactosaminyltransferase-like protein 5 (GALNTL5), also described as pp-GalNAc-T19 (Peng et al., 2010) or GalNAc-T20 (Raman et al., 2012), belongs to the polypeptide $\mathrm{N}$-acetylgalactosaminetransferase (pp-GalNAc-T) gene family. GALNTL5 possesses the highly conserved catalytic domains of pp-GalNAc-T but uniquely truncates the conserved lectin domain at the C-terminus. The GALNTL5 mRNA is expressed exclusively in the testes, mainly in the cytoplasm of round and elongated spermatids during spermiogenesis and not in the outermost cells of the seminiferous tubules, which contain spermatogonia and somatic Sertoli cells (Takasaki et al., 2014). GALNTL5 is indispensable for the formation of mature sperm and may have a unique role in mammalian spermiogenesis. In round spermatids, GALNTL5 is distributed in the juxtanuclear space and not in the acrosomal vesicles. In elongated spermatids, GALNTL5 is highly localized in the acroplaxome, which is the region between the developing acrosome and nucleus (Kierszenbaum et al., 2003). During differentiation, the signals are also weakly detected in the transient manchette containing microtubules (Kierszenbaum and Tres, 2004). In epididymal spermatozoa, GALNTL5 is concentrated to the neck region around the head-tail coupling apparatus and is only weakly detected in the midpiece. Notably, GALNTL5 also temporarily localizes in the acroplaxome and manchette, which is a transient microtubular/actin structure, during spermatid elongation and finally accumulates in the neck region around the head-tail coupling apparatus of mature spermatozoa. These findings lend strong evidence for GALNTL5 being involved in spermiogenesis (Takasaki et al., 2014).

Several studies have indicated that the protein components involved in vesicle cargo transport and the ubiquitin-proteasome system alter the distribution of differentiating spermatids through a route similar to that of GALNTL5 (Kierszenbaum et al., 2011). GALNTL5 participates in the transport of vesicle cargos and in the ubiquitin-proteasome system in the region of the head-tail coupling apparatus for mature sperm formation. In the spermatozoa of heterozygous mutant mice, some researchers observed impairment of protein loadings into acrosomes and aberrant localization of the ubiquitin-proteasome system. Moreover, colocalization of GALNTL5 signals with UBC3B, an E2 ubiquitin conjugating enzyme similar to $\mathrm{UBC} 3 / \mathrm{CDC} 34$, and ubiquitin signals was observed in the ubiquitin-proteasome system in the neck region of spermatozoa from wild-type mice (Takasaki et al., 2014).

Spermatogenesis is a very intricate and tightly regulated process in which germ cells at each stage, including the proliferative phase (spermatogonia), meiotic phase (spermatocytes), and differentiation or spermiogenic phase (spermatids), are produced in a coordinated manner (Eddy, 2002). Different stages of germ cells possess distinct gene expression levels, which are regulated at transcriptional (Goldberg, 1996), post-transcriptional (Wiszniak et al., 2011), translational (Baker and Fuller, 2007), and post-translational (Lalancette et al., 2006) levels.

Alternative splicing (AS) is an essential function by which post-transcriptional gene regulation occurs in cells (Ochs et al., 2012), with no exception to intrinsic regulation in 
germ cells. This phenomenon is universal to numerous protein-coding genes of multicellular organisms. In general, alternatively spliced exons have 5'- and 3'-splice site motifs that differ significantly from the normal motifs. In addition to these splice sites, exons are defined by cis-acting regulatory elements, which have been divided into four functional categories: exonic splicing enhancers, exonic splicing silencers, intronic splicing enhancers (also known as intronic activators of splicing), and intronic splicing silencers. These cis-acting elements interact directly or indirectly with trans-acting activators or repressors of splicing (Keren et al., 2010). In a novel splice variant PLCz1-sv1 in Chinese Holstein bulls, the AS pattern of alternating 5 '-splice sites is characterized in the testis tissues (Ju et al., 2014). Thus, AS may be an additional mechanism by which the diverse physiologic functions of the GALNTL5 gene are regulated. To date, little is known about GALNTL5 mRNA expression in bull, and splice variants of bovine GALNTL5 have not yet been characterized. In the present study, we identified GALNTL5 splice variants in Chinese Holstein bull testis tissue.

\section{MATERIAL AND METHODS}

\section{Collection of tissue samples}

Tissue samples, including heart, liver, spleen, lung, kidney, and testis, were collected from 13 randomly culled Chinese Holstein cattle, including four calves and nine adult bulls. Of these samples, all collected at slaughterhouses, two calves and five adult bulls came from the Shandong OX Bio-Technology Co., Ltd., whereas all other samples came from the Duoshi Liuyingzi Commercial Slaughter Farm in Jinan, Shandong Province, China. Tissue samples were obtained immediately after slaughter, snap frozen in liquid nitrogen, and transported to the laboratory. The tissue samples were kept frozen in liquid nitrogen until RNA isolation and cDNA synthesis for the relative expression analysis of the GALNTL5 gene and identification of splice variants.

\section{Identification of the splice variants of the bovine GALNTL5 gene}

To investigate potential splice variants of the bovine GALNTL5 gene, one pair of specific primers GALNTL5-cDNA (Table 1) was designed using Primer Premier v. 5.0 software. These primers amplified the full-length transcript and splice variants, according to the bovine GALNTL5 reference sequence (GenBank accession No. NM_001076441.1). Total RNA was isolated from the 13 bovine tissue samples using an RNAsimple Total RNA Kit (Tiangen, Beijing, China), following the manufacturer instructions. The RNA concentration was measured using a Biophotometer (Implen, Germany) and the RNA integrity was determined via electrophoresis on $1 \%$ agarose gel. Then, a RevertAid ${ }^{\mathrm{TM}}$ First Strand cDNA Synthesis Kit (Fermentas, Canada) was used to convert $\sim 1 \mu \mathrm{g}$ RNA from each sample into cDNA according to the manufacturer specifications. A polymerase chain reaction (PCR) was performed with an initial denaturation step at $94^{\circ} \mathrm{C}$ for $5 \mathrm{~min}$, followed by 35 cycles at $94^{\circ} \mathrm{C}$ for $30 \mathrm{~s}, 59^{\circ} \mathrm{C}$ for $30 \mathrm{~s}$, and $72^{\circ} \mathrm{C}$ for $1.5 \mathrm{~min}$, with a final extension at $72^{\circ} \mathrm{C}$ for $10 \mathrm{~min}$. Splice variants were identified based on the method used by Ju et al. (2012). In brief, DNA bands were separated using $1.5 \%$ agarose gel electrophoresis and purified using a GenClean kit (Cwbiochem, Beijing, China). The purified products were then cloned into the pEASY-T3 vector (TaKaRa, Dalian, China) and transformed into Escherichia coli DH5 $\alpha$ cells. The cells 
were subsequently propagated in LB medium overnight at $37^{\circ} \mathrm{C}$. The positive clones were randomly selected.

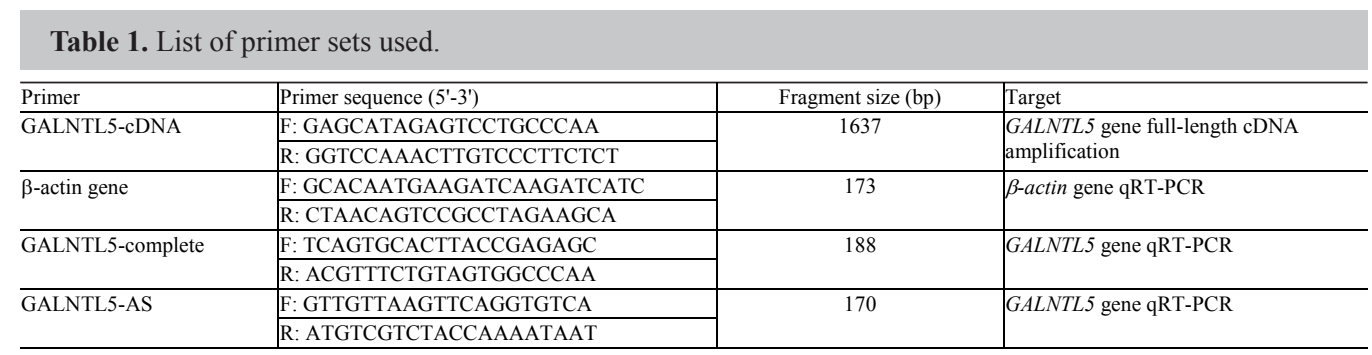

\section{Quantification of abundance of GALNTL5 transcript variants}

Quantitative real-time PCR (qRT-PCR) was performed to determine the relative mRNA expression of the GALNTL5 splice variants in adult bull and calf testes. We designed the corresponding specific PCR primers to determine the differential expression between GALNTL5-complete and GALNTL5-AS (Table 1). The qRT-PCR analysis was performed in a $20-\mu \mathrm{L}$ mixture containing $2 \mu \mathrm{L}$ cDNA, $0.4 \mu \mathrm{M}$ sense and antisense primers, $6.4 \mu \mathrm{L} \mathrm{dH_{2 }} \mathrm{O}$, and $10.0 \mu \mathrm{L} 2 \mathrm{X}$ SYBR $^{\circledR}$ Premix Ex Taq ${ }^{\mathrm{TM}}$ (TaKaRa). The qRT-PCR was performed under the following conditions: pre-incubation at $95^{\circ} \mathrm{C}$ for $30 \mathrm{~s}, 40$ amplification cycles of $5 \mathrm{~s}$ at $95^{\circ} \mathrm{C}$ followed by $60^{\circ} \mathrm{C}$ for $30 \mathrm{~s}$. The conditions for the last stage of the melting curve were as follows: $95^{\circ} \mathrm{C}$ for $5 \mathrm{~s}, 60^{\circ} \mathrm{C}$ for $1 \mathrm{~min}$, and $95^{\circ} \mathrm{C}$ for $15 \mathrm{~s}$. The PCR was monitored using a Light-Cycler ${ }^{\mathbb{B}}$ 480 II Real-time PCR system (Roche, Switzerland). In addition, the bovine housekeeping gene $\beta$-actin (GenBank accession No. NM_173979.3) was used as an internal control, to normalize alternatively spliced transcription values. Each sample was run in triplicate.

\section{Data mining}

AS detection and sequence analysis were conducted using the BLAST online search resource (http://blast.ncbi.nlm.nih.gov) and DNAMAN software package (v. 6.0, Lynnon Biosoft, Quebec, Canada). The ORF Finder in NCBI was used to identify the open reading frame. Cross-species genomic comparisons and functional domain localization within GALNTL5 were conducted using the Ensembl database (www.Ensembl.org). The secondary structure of retained introns was predicted using the RNAfold web server (http:// rna.tbi.univie.ac.at/cgi-bin/RNAfold.cgi). The 3D structure of the putative GALNTL5 protein was analyzed using SWISS-MODEL (http://swissmodel.expasy.org/workspace/ index.php?func $=$ modelling_simple 1 ).

\section{Statistical analyses}

The relative quantification of GALNTL5 mRNA expression was calculated using the standard curve-based method for RT-PCR (Larionov et al., 2005). The analyses were performed using SAS v. 9.3 statistical software (SAS Institute Inc., Cary, NC, USA). Data are presented as means or mean \pm SEM. Statistical significance $(\mathrm{P}<0.05)$ for gene expression was tested using a Student's $t$-test. 


\section{RESULTS}

\section{Identification of the bovine $G A L N T L 5$ splice variants}

To investigate the possible splicing variants of the bovine GALNTL5 gene, RT-PCR was performed on bovine heart, liver, spleen, lung, kidney, and testis tissue samples. We only found two main bands in the testis (Figure 1A), after amplifying the full-length GALNTL5 transcript with the primers specific for GALNTL5-cDNA (Table 1) as templates. The two bands of 1637 and 1516 bp were purified, subcloned, and sequenced. When comparing the sequence obtained from each sample with the bovine GALNTL5 genomic (GenBank accession No.AC_000161.1) and RNA sequence (GenBank accession No. NM_001076441.1), we found a new splice variant in the testis named GALNTL5-AS (Figure 1C) lacking exon 2 (Figure 1B). This sequence was submitted to NCBI (GenBank Accession No. KT763324).

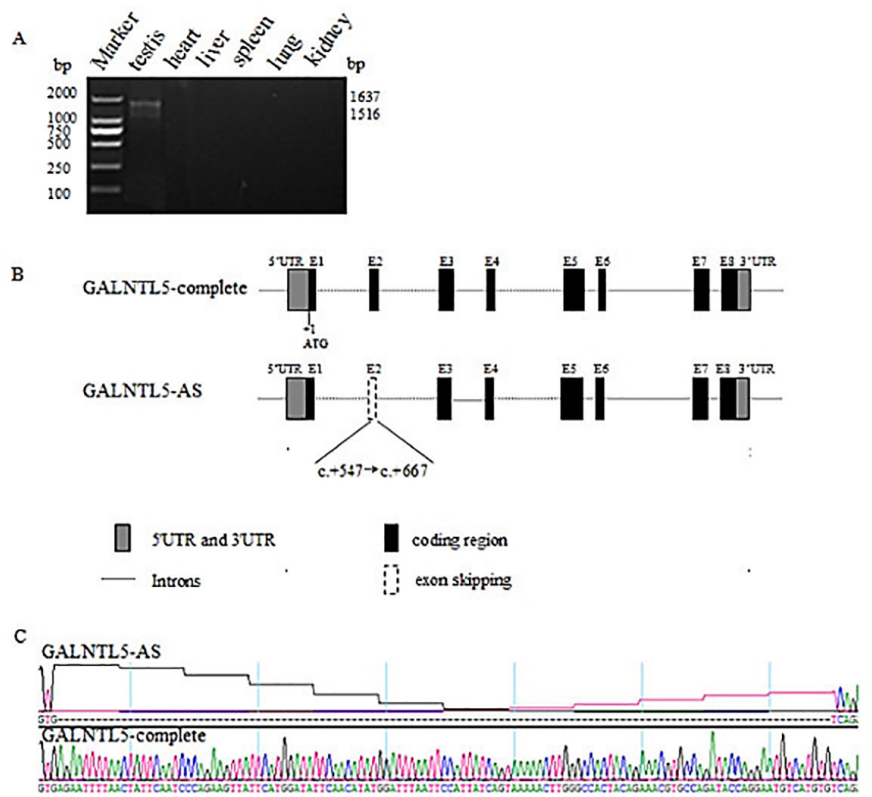

Figure 1. Expression of GALNTL5 mRNA and identification of bovine GALNTL5 splice variants. A. RT-PCR analysis of GALNTL5 transcript expression in various adult bull tissues. B. Diagrammatic representation of the genomic structure of bovine GALNTL5 and alternative splicing pattern of GALNTL5 transcripts. C. Sequencing results of GALNTL5-complete and GALNTL5-AS.

\section{GALNTL5-complete and GALNTL5-AS quantification}

Selective amplification of GALNTL5-complete and GALNTL5-AS was achieved by RT-PCR using specific primers (Figure 2A). We then performed relative quantification of the GALNTL5-complete and GALNTL5-AS transcripts in adult bull and calf testes, using RT-PCR amplification with conditions optimized for each of the isoforms of GALNTL5. As demonstrated in Figure 2B, the expression of both transcripts displayed notably higher levels in adult bull testes compared to in calf testes $(\mathrm{P}<0.05)$. In addition, in adult bull testes, a 
higher expression of GALNTL5-complete than GALNTL5-AS was observed. In contrast, the opposite was observed in calf testes where GALNTL5-AS manifested a higher expression than GALNTL5-complete. These findings were consistent with the RT-PCR results in the current study, suggesting that GALNTL5 mRNA displayed signs of varying temporal expression.

adult bull testis

A

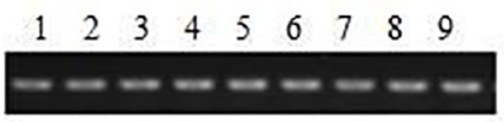

GALNTL5-complete

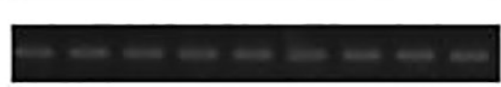

GALNTL5-AS

$\beta$-actin
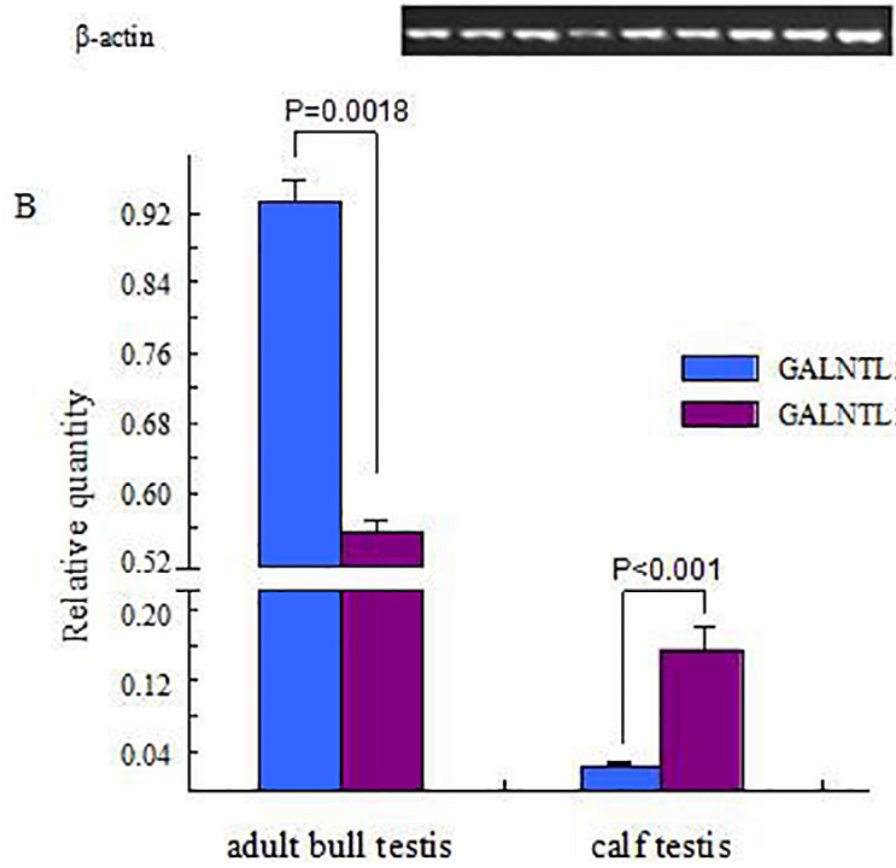

Figure 2. Relative expression of the GALNTL5 gene. A. Expression of the GALNTL5-complete and GALNTL5-AS transcripts in nine adult bull testes using RT-PCR. B. Relative expression of GALNTL5-complete and GALNTL5AS in testis tissue of adult bulls and calves using qRT-PCR. The vertical bars represent standard errors.

\section{In silico analysis of $G A L N T L 5$ mRNA and protein}

The open reading frame of GALNTL5-AS with 122 amino acids was predicted by ORF Finder in NCBI. Only the E1 and partial E3 domain were transcribed. The typical domain of the bovine GALNTL5 protein was analyzed utilizing the Ensembl database and protein blast. The structure is presented in Figure 3A. The GALNTL5-AS transcript had a deletion of the glycosyltransferase 1 (GT1) and Gal/GalNAc-T motifs compared with GALNTL5-complete. The 3D structure of the putative GALNTL5 protein also confirmed this finding (Figure 3B). 
Cross-species genomic comparisons indicated that the bovine GALNTL5 protein shared low sequence identity with human (67\%) and murine (60\%), but GALNTL5 was conserved in the conserved domains of human, murine, and other bovine species (Figure 4).

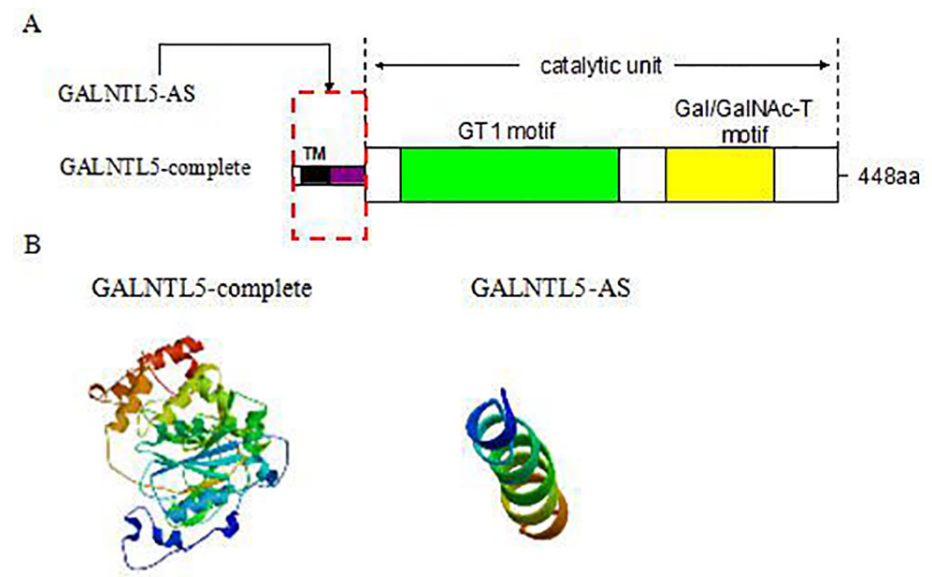

Figure 3. Sketch map of the bovine GALNTL5 protein. A. Linear map of the bovine GALNTL5 polypeptide shows the domain organization. The red box represents the diagram of GALNTL5-AS. B. 3D structures of two putative GALNTL5 proteins produced using the SWISS-MODEL software.

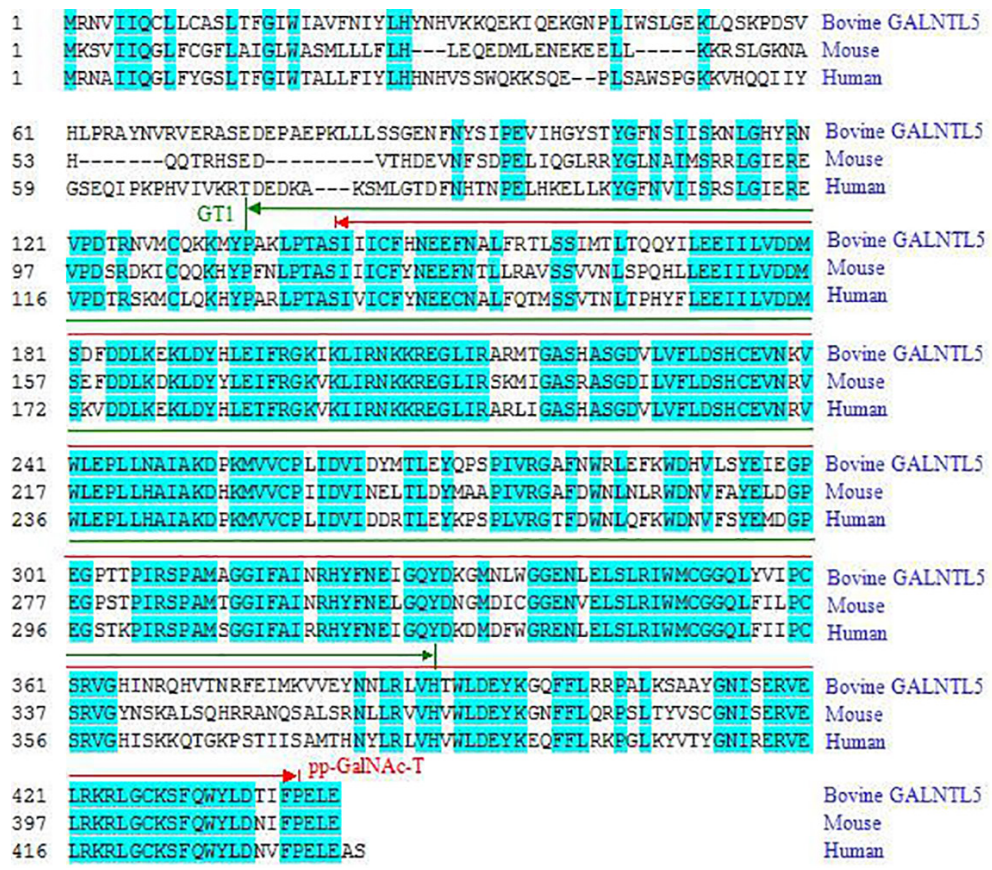

Figure 4. Comparison of the bovine, murine, and human GALNTL5 amino acid sequences. The bovine GALNTL5 amino acid sequence (NCBI: NP 001069909.1) was aligned to the human (NCBI: NP 660335.2) and murine (NCBI: NP_080725.2) sequences using BLAST. Identical amino acid residues within the three species are highlighted in blue. 


\section{DISCUSSION}

Functionally mature sperm must be motile and have a fully formed acrosome for normal fertilization in mammals. GALNTL5 is a functional molecule indispensable for mammalian sperm formation. The GALNTL5 mRNA is mostly expressed in the testes. GALNTL5 temporarily localizes in the acroplaxome and manchette, finally accumulates in the neck region of mature spermatozoa. Takasaki et al. (2014) found a GALNTL5 heterozygous mutation in mice. This mutation attenuated the amounts of glycolytic enzymes required for motility, including acrosin, N-ethylmaleimide-sensitive factor, and testicular angiotensinconverting enzyme (Takasaki et al., 2014). The mutation also disrupted protein loadings into acrosomes and caused aberrant localization of the ubiquitin-proteasome system in the neck region of the spermatozoa, which consist of common cytoplasmic constituents shared among the haploid spermatids through the intercellular bridges (Takasaki et al., 2014). In the present study, we found that the expression of GALNTL5 mRNA was mostly restricted to the bovine testis tissue, as shown by RT-PCR. However, GALNTL5 in adult bull displayed higher expression than that in calf. Therefore, we speculate that the GALNTL5 gene is also an vital functional molecule during bull sperm formation.

AS is ubiquitous in bacteria and humans (Keren et al., 2010). It influences the coding sequence, leading to the production of diverse proteins (Graveley, 2001; Swami, 2009). New high-throughput sequencing technology has revealed that $90 \%$ of human genes are involved in AS (Wang et al., 2008). Takasaki et al. (2014) also found that the human GALNTL5 gene had one heterozygous nucleotide deletion at the sixth exon resulting in a stop codon of the sixth exon, which caused a frame shift in the asthenozoospermic sperm of human. This mutation formed a new splice variant that decreased the amounts of GALNTL5 and contributed to asthenozoospermia. In the present study, a new GALNTL5 splice variant named GALNTL5-AS was identified in Chinese Holstein bulls through cloning of GALNTL5 mRNA, which showed a skipping of the second exon. In addition, in silico analysis showed that the GALNTL5-AS transcript only encoded for 122 amino acids. The transcript had lost its GT1 and Gal/GalNAc-T motifs and may ultimately result in disruption of the spermatogenetic function compared with the primary transcript GALNTL5-complete. GALNTL5 is classified as a member of the pp-GalNAc-T family, because it possesses the highly conserved catalytic domains of pp-GalNAc-T but uniquely lacks the conserved lectin domain at the C-terminus. The pp-GalNAc-T family belongs to the type II membrane proteins (Roth et al., 1994; Röttger et al., 1998). Their fundamental structure encompasses a short cytoplasmic tail; a single transmembrane anchoring domain; a stem region with variable length; a catalytic domain with GT1 and Gal/GalNAc-T motifs, which are the putative binding sites for donor and acceptor substrate, respectively; and a C-terminus ricintype lectin domain (Hazes, 1996). Thus, since GALNTL5-AS is lacking the GT1 and Gal/ GalNAc-T motifs, it may affect bovine sperm formation.

In conclusion, a novel GALNTL5 splice variant named GALNTL5-AS was identified, and different splicing patterns were detected in bovine testes. We detected expression levels of two transcripts in adult bull and calf testes and found that the GALNTL5-AS may be involved in spermatogenesis. This study may ultimately facilitate a better understanding of the GALNTL5 gene structure and function. However, the biologic significance of the GALNTL5-AS transcript requires further exploration, including more replicated studies. 


\section{Conflicts of interest}

The authors declare no conflict of interest.

\section{ACKNOWLEDGMENTS}

Research supported by grants from the National Natural Science Foundation of China (\#31371255,\#31271328, and \#31401050), the Major Project of National Transgenics in China (\#2014ZX08007-001), the National Cow Industrial Technology System Program (\#CARS37), the Cow Innovation Team of the Shandong Province Modern Agricultural Industry Technology System (\#SDAIT-12-011-02), the Natural Science Foundation of Shandong Province (\#ZR2013CQ035), and the Agricultural Well-bred Program of Shandong Province (\#2014LZ).

\section{REFERENCES}

Baker CC and Fuller MT (2007). Translational control of meiotic cell cycle progression and spermatid differentiation in male germ cells by a novel eIF4G homolog. Development 134: 2863-2869. http://dx.doi.org/10.1242/dev.003764

Eddy EM (2002). Male germ cell gene expression. Recent Prog. Horm. Res. 57: 103-128. http://dx.doi.org/10.1210/ rp. 57.1 .103

Goldberg E (1996). Transcriptional regulatory strategies in male germ cells. J. Androl. 17: 628-632.

Graveley BR (2001). Alternative splicing: increasing diversity in the proteomic world. Trends Genet. 17: 100-107. http:// dx.doi.org/10.1016/S0168-9525(00)02176-4

Hazes B (1996). The (QxW)3 domain: a flexible lectin scaffold. Protein Sci. 5: 1490-1501. http://dx.doi.org/10.1002/ pro. 5560050805

Ju Z, Wang C, Li Q, Hou M, et al. (2012). Alternative splicing and mRNA expression analysis of bovine SLAMF7 gene in healthy and mastitis mammary tissues. Mol. Biol. Rep. 39: 4155-4161. http://dx.doi.org/10.1007/s11033-011-1198-z

Ju ZH, Pan Q, Zhang Y, Huang JM, et al. (2014). Identification and characterization of a novel splice variant of the PLC $\zeta 1$ gene in bull testis tissues. Genet. Mol. Res. 13: 9899-9909. http://dx.doi.org/10.4238/2014.November.27.18

Keren H, Lev-Maor G and Ast G (2010). Alternative splicing and evolution: diversification, exon definition and function. Nat. Rev. Genet. 11: 345-355. http://dx.doi.org/10.1038/nrg2776

Kierszenbaum AL and Tres LL (2004). The acrosome-acroplaxome-manchette complex and the shaping of the spermatid head. Arch. Histol. Cytol. 67: 271-284. http://dx.doi.org/10.1679/aohc.67.271

Kierszenbaum AL, Rivkin E and Tres LL (2003). Acroplaxome, an F-actin-keratin-containing plate, anchors the acrosome to the nucleus during shaping of the spermatid head. Mol. Biol. Cell 14: 4628-4640. http://dx.doi.org/10.1091/mbc. E03-04-0226

Kierszenbaum AL, Rivkin E and Tres LL (2011). Cytoskeletal track selection during cargo transport in spermatids is relevant to male fertility. Spermatogenesis 1: 221-230. http://dx.doi.org/10.4161/spmg.1.3.18018

Lalancette C, Bordeleau LJ, Faure RL and Leclerc P (2006). Bull testicular haploid germ cells express a messenger encoding for a truncated form of the protein tyrosine kinase HCK. Mol. Reprod. Dev. 73: 520-530. http://dx.doi. org $/ 10.1002 / \mathrm{mrd} .20422$

Larionov A, Krause A and Miller W (2005). A standard curve based method for relative real time PCR data processing. BMC Bioinformatics 6: 62. http://dx.doi.org/10.1186/1471-2105-6-62

Ochs MJ, Sorg BL, Pufahl L, Grez M, et al. (2012). Post-transcriptional regulation of 5-lipoxygenase mRNA expression via alternative splicing and nonsense-mediated mRNA decay. PLoS One 7: e31363. http://dx.doi.org/10.1371/ journal.pone. 0031363

Peng C, Togayachi A, Kwon YD, Xie C, et al. (2010). Identification of a novel human UDP-GalNAc transferase with unique catalytic activity and expression profile. Biochem. Biophys. Res. Commun. 402: 680-686. http://dx.doi. org/10.1016/j.bbrc.2010.10.084

Raman J, Guan Y, Perrine CL, Gerken TA, et al. (2012). UDP-N-acetyl- $\alpha$-D-galactosamine:polypeptide $\mathrm{N}$-acetylgalactosaminyltransferases: completion of the family tree. Glycobiology 22: 768-777. http://dx.doi. org/10.1093/glycob/cwr183 
Roth J, Wang Y, Eckhardt AE and Hill RL (1994). Subcellular localization of the UDP-N-acetyl-D-galactosamine: polypeptide $\mathrm{N}$-acetylgalactosaminyltransferase-mediated O-glycosylation reaction in the submaxillary gland. Proc. Natl. Acad. Sci. USA 91: 8935-8939. http://dx.doi.org/10.1073/pnas.91.19.8935

Röttger S, White J, Wandall HH, Olivo JC, et al. (1998). Localization of three human polypeptide GalNAc-transferases in HeLa cells suggests initiation of O-linked glycosylation throughout the Golgi apparatus. J. Cell Sci. 111: 45-60.

Swami M (2009). Alternative splicing: deciding between the alternatives. Nat. Rev. Genet. 10: 71. http://dx.doi. org/10.1038/nrg2532

Takasaki N, Tachibana K, Ogasawara S, Matsuzaki H, et al. (2014). A heterozygous mutation of GALNTL5 affects male infertility with impairment of sperm motility. Proc. Natl. Acad. Sci. USA 111: 1120-1125. http://dx.doi.org/10.1073/ pnas. 1310777111

Wang ET, Sandberg R, Luo S, Khrebtukova I, et al. (2008). Alternative isoform regulation in human tissue transcriptomes. Nature 456: 470-476. http://dx.doi.org/10.1038/nature07509

Wiszniak SE, Dredge BK and Jensen KB (2011). HuB (elav12) mRNA is restricted to the germ cells by post-transcriptional mechanisms including stabilisation of the message by DAZL. PLoS One 6: e20773. http://dx.doi.org/10.1371/ journal.pone.0020773 